\title{
Identifying Adolescent Problem Gambling Using Latent Variable Techniques
}

\author{
Emil Frasheri
}

PhD candidate, Department of Management, Faculty of Economy, Fan S. Noli University of Korça - Albania

Besa SHAHINI

Prof. Dr., Department of Applied Statistics and Informatics, Faculty of Economy, University of Tirana- Albania

\begin{abstract}
Recently disordered gambling is reclassified as an addictive disorder which inter alia affects a little but significant proportion of adolescents. The aim of this study is to identify and assess different levels of gambling severity among adolescent gamblers $(\mathrm{N}=1157)$ from middle and high schools of Korca region, utilizing a crosssectional design and self-report questionnaire. Using Exploratory, Parallel, Reliability and Confirmatory Analysis, the PGSI measurement instrument, designed for these purposes, resulted to be an appropriate unidimensional screening tool of adolescent disordered gambling in terms of psychometric properties. A traditional Latent Class Analysis using the nine PGSI-items as indicators is performed to identify and predict subtypes of adolescent gamblers, classifying them into latent classes based on their problem gambling severity levels. The inclusion of three covariates related to adolescent gambling problems into Latent Class Model improved the model, helping us to better understand the latent structure.
\end{abstract}

Keywords: adolescents, disordered gambling, latent variables, measurement instruments.

\section{Introduction}

Problem gambling is being viewed increasingly as a behavioural addiction and has been re-classified from an impulse control disorder to an addictive disorder in the Diagnostic and Statistical Manual of Mental Disorders, Fifth Edition (APA, 2103). Problem and pathological gambling are recognized as being major societal problem, with millions of individuals suffering from them. While disordered and pathological gambling has been primarly thought of as an adult problem, there have been increased research efforts to examine the prevalence and underlying factors associated with problems among adolescents. The rates of problem gambling in children and adolescents are nearly two times higher than for adults ( 3 $8 \%$ ), however, it is estimated that $10-14 \%$ of youth exhibit behaviors that place them at increased risk for developing gambling problems (Petry, 2005). Shaffer and Hall (1999) provided the semantic architecture of problem gambling selecting the phrase disordered gambling. This must be conceptualized as a wide range of gradual shifting from regulated gambling to problem or pathological gambling. To facilitate the estimation of prevalence and the understanding of disordered gambling, researchers have divided this continuum in distinct categories. The majority of people gamble with little or no adverse consequences. These people are classified as Level1 gamblers ("non-problem" gamblers). However gambling is associated with meaningful negative effects for certain segments of the population. The people who experience some negative consequences are the Level 2 gamblers ("at-risk" gamblers), whereas individuals with the most serious of these consequences are Level 3 gamblers, which are refered as "problem" or "disordered" gamblers. Level 2 gamblers represent people who may be moving in either of two directions: some Level 2 gamblers are moving toward an increasingly disordered state, while others are moving toward Level 1 gambling. Studying all gamblers, considering problem gambling severity levels, may be helpful to better understand gambling related problems. As a result, it is important to build a predictive or structural model for class membership, underlying problem gambling severity levels. Identifying groups based on similar behavioral characteristics and using appropriate methods to model gambling behavior may be particularly useful. In a more explanatory study, one may wish to build a predictive or structural model for class membership whereas in a more descriptive study the aim would be to simply profile the latent classes by investigating their association with external variables (covariates) and to examine if some risk or protective factors of problem gambling among adolescents influence gambling problems differently across classes. 


\section{Correlates of adolescent disordered gambling \\ Gender}

Male gender has continuously been linked as a risk factor for disordered gambling (Blanco et al., 2006). Males can gamble longer than females before their gambling develops as disordered (Potenza et al., 2006; Ibanez et al., 2003; Ladd \& Petry, 2002; Tavares et al., 2001). Males gamble more than females, particularly at more severe level (Shaffer et al., 1999; Potenza et al., 2006). Adolescent males are more involved in gambling than females, in terms of both frequency and expenditure, and that they experience more gambling related problems than females (Buchta, 1995; Griffiths, 1991; Chiu \& Storm, 2010; Derevensky et al., 2010). In a study of middle and high school students, males were found to be almost six times more likely than females to be identified as having a gambling problem and twice as likely to be classified as at-risk gamblers, endorsing a number of criteria for gambling problems (Dickson et al., 2008). Pathological gamblers are thought to be 5 to 10 times more likely than recreational gamblers to have a co-morbid addiction (drug, alcohol) (Daghestani et al., 1996).

Substance use

The links between gambling and substance use have also been found in many studies (Barnes et al., 2009; Goldstein et al., 2009; Jackson et al., 2008). Disordered gambling and substance abuse are more severe when they co-occurs compared to those with only one of the disorders (Petry, 2000). Clearly, data on adolescent substance use and gambling indicate that these behaviors tend to co-occur in youth suggesting that substance use should be viewed as a warning sign for comorbid gambling problems and vice versa.

Peer influence

Adolescent problem gamblers tend to have peers who gamble (Donati et al., 2012) and those peers often have gambling problems (Hardoon \& Derevensky, 2002). Having peers who gamble, especially peers who gamble excessively, has consistently been found to be a risk factor for youth gambling problems (Jacobs, 2000). Gupta and Derevensky (2000) indicated that, having friends who engage in any addictive behavior, and not just gambling, poses a significant risk factor.

\section{STATISTICAL METHODS}

\section{Exploratory Factor Analysis (EFA) and Confirmatory Factor Analysis (CFA).}

Factor Analysis is a statistical technique applied to a set of variables, discovering which variables in the set form coherent subsets that are relatively independent to one another. Variables correlated with one another but largely independent of other subsets are combined into factors. The purpose of Exploratory Factor Analysis is to determine the number of factors that are needed to explain the correlations among a set of observed variables. This technique help us to understand the structure of the set of observed variables, reducing the data set to a more manageable size while retaining as much of the original information as possible. EFA is an "exploratory" analysis because no a priori restrictions are placed on the pattern of relationships between the observed and latent variables. This is a key difference between EFA and CFA. In CFA, the researcher must specify in advance several key aspects of the factor model such as the number of factors and patterns of indicator-factor loadings. These techniques often rely on Maximum Likelihood estimation (ML) which helps us to evaluate how well the factor solution is able to reproduce the relationships among the observed variables in the sample. This is very helpful for determining the appropriate number of factors. EFA is often used as a precursor to CFA in scale development and construct validation. The process of deciding how many factors to keep is called extraction. We retain only factors with large eigenvalues $(\lambda>1)$. An additional technique is Horn's Parallel Analysis (Horn, 1965). Parallel Analysis compares the random data eigenvalues at the $95^{\text {th }}$ percentile with the eigenvalues of the polychoric matrix. Only those eigenvalues that exceed the corresponding values from the random data set are retained. CFA is much more sophisticated technique used in the advanced stages of the research process where variables are carefully and specifically chosen to reveal underlying processes.

\section{Latent Class Analysis (LCA)}

A statistical technique that can be used to characterize patterns of gambling involvement is Latent Class Analysis (LCA) (Lazarsfeld \& Henry, 1968; Goodman, 1974; McCutcheon, 1987; Vermunt \& Magidson, 2004; Collins \& Lanza, 2010). Latent Class Analysis (LCA) is a statistical method used to identify subtypes of related cases using a set of categorical or continuous observed variables. These subtypes are referred to as latent classes. The classes are latent because the subtypes are not directly observed; rather, they are inferred from the multiple observed variables or indicators. When categorical data are used, the latent class model has the advantage of making no assumptions about the distributions of 
the indicators other than that of local independence. LCA application follows a three step- process: 1) a LC model is built for a set of response variables, 2) subjects are assigned to latent classes based on their posterior class membership probabilities, and 3 ) the association between the assigned class membership and external variables is investigated using simple cross-tabulations or multinomial logistic regression analysis (Vermunt, 2010). LCA has two main quantifiable issues: a) Latent class probabilities and b) conditional probabilities for each class. Latent class probabilities describe the distribution of classes of the latent variable within which the observed measures are (locally) independent of one another. Conditional probabilities represent the probability of an individual in a given class of the latent variable being at a particular level of the observed variables. The goal of LCA is to determine the smallest number of latent classes that is sufficient to explain away the relationships among observed indicators. In order to obtain improved description and prediction of the latent variable(s), multinomial or logistic regression models are used to express these probabilities as a function of one or more exogenous variables called covariates (Dayton \& Macready, 1988). The inclusion of covariates into mixture models allow us to examine relationships of mixture classes and auxiliary information, to understand how different classes relate to risk and protective factors or to examine differences in demographics across the classes. Controversary, including covariates in mixture models, latent class variable may have an undesirable shift in the sense that it is no longer measured simply by the original latent class indicator variables but now it is also measured by the auxiliary variables (Asparouhov \& Muthèn, 2014). However, a recent study (Wurpts \& geiser, 2014) suggested that researchers can in general feel comfortable using a larger set of indicators and adding theoretically meaningful covariates to the model.

\section{Assessing Model Fit}

Fit indices are often used to supplement the $\chi^{2}$ test to evaluate the acceptability of latent variable models. The most commonly used information criteria (IC) in different studies are: Comparative Fit Index (CFI) (Bentler, 1988) and Tucker Lewis Index (TLI) (Tucker \& Lewis, 1973) which are incremental fit indices, measuring the improvement of fit by comparing the hypothesized model with a more restricted baseline model. Values can range from 0-1. For these indices, values above .90 indicate reasonable fit. Values above .95 indicate good model fit. CFI has smaller sampling variability than TLI. The Root Mean Square Error of Approximation (RMSEA) estimates the lack of fit in a model compared to a perfect (saturated) model. Browne and Cudeck (1993) suggested that RMSEA values larger than 0.1 are indicative of poor-fitting models, values in the range of 0.05 to 0.08 are indicative of fair fit and values less than 0.05 are indicative of close fit. A cut-off value of RMSEA close to 0.06 has been recommended. SRMR and WRMR are residual-based fit indices that measure the (weighted) average differences between the sample and estimated population variances and covariances. SRMR-the standardized root mean-square residual (Joreskog \& Sorbom, 1981) is an absolute measure of fit. It is defined as the standardized difference between the observed correlation and the predicted correlation. A cut-off value close to 0.08 has been recommended for SRMR. WRMR- Weighted Root-mean-square Residual (Muthèn \& Muthèn, MPlus User's Guide, $2^{\text {nd }}$ Version 1998-2001) is suitable for models where sample statistics have widely disparate variances and when sample statistics are on different scales such as in models with mean and/or threshold structures. It is also suitable with non-normal outcomes. WRMR $\leq 1.0$ can be used to identify good simple models when $N \geq 500$. Some other important model fit indices are the penalized information criteria AIC, BIC, and adjusted BIC. The Akaike Information Criterion (AIC) (Akaike, 1987) is defined as:

$A I C=-2 \log L+2 p$ (Eq.1)

where $p$ is the number of free model parameters. The Bayesian Information Criterion BIC (Schwartz, 1978) is defined as: $\mathrm{BIC}=-2 \log \mathrm{L}+\mathrm{p} \log (\mathrm{n})($ Eq. 2$)$

The adjusted BIC (SABIC) (Sclove, 1987) replaces the sample size $n$ in the BIC equation above with $n^{*}$ : $\mathrm{n}^{*}=(\mathrm{n}+2) / 24$. (Eq. 3)

A smaller AIC and BIC for a particular model suggests that the trade-off between fit and parsimony is preferable. AIC is not a good indicator for class enumeration for LCA models with categorical outcomes (Yang, 2006). The BIC and the adjusted BIC are comparatively better indicators of the number of classes than the AIC. Comparing across all the models and sample sizes, there seems to be strong evidence that the BIC is the best of the Information Criteria considered (Nylund et al., 2007; Collins et al., 1993; Hagenaars \& McCutcheon, 2002; Magidson \& Vermunt, 2004; Jedidi et al., 1997). The Lo-Mendell Rubin (LMR) adjusted (LRT) test is able to distinguish between the k-1 and k class models, comparing the improvement in fit between these neighboring class models and providing a $p$-value that can be used to determine if there is a statistically significant improvement in fit for the inclusion of one more class. A low p-value rejects the $\mathrm{k}-1$ class model in favor of the $\mathrm{k}$ class model. Mplus reports the relative entropy of the model, which is a rescaled 
version of entropy. The relative entropy is defined on $[0,1]$, with values near one indicating high certainty in classification and values near zero indicating low certainty.

\section{DATA ANALYSIS}

The study included 1157 participants (53.5\% females, $46.5 \%$ males) from middle and high schools of Korça region, ranged from 18 to 23 years old. The survey was conducted between June and July, 2015. Data were collected using a self-reported questionnaire. We focused only on respondents who had played at least ones during the last 12 months. Participants were given a questionnaire during regular class time assessing their past gambling history, frequency of gambling behavior, other addictive behaviours, and PGSI items. The total time required for completion of the questionnaire was approximately 20 minutes.

\section{Measures}

It is used SPSS and Mplus software packages to analyze our data. Missing data are assumed to be "missing at random", and Mplus used all data available to estimate the model under the MCAR mechanism. Gambling problems among students were measured using the nine-item questionnaire of the Problem Gambling Severity Index (PGSI) measurement instrument. Among a lot of measurement instruments, the consensus in the literature is that the PGSI (Ferris \& Wynne, 2001 ) is the most appropriate measure of disordered gambling in terms of psychometric properties (Jackson et al., 2009; McMillen \& Wenzel, 2006; Svetieva \& Walker, 2008). This nine-item self-report instrument was designed to measure a single problem gambling construct. Four of the nine items assess problem gambling behaviors: Have you bet more than you could really afford to lose? (Bet); Have you needed to gamble with larger amounts of money to get the same feeling of excitement? (Tolerance); When you gambled, did you go back another day to try to win back the money you lost? (Chase); Have you borrowed money or sold anything to get money to gamble? (Borrowed). The other five items assess adverse consequences of gambling: Have you felt that you might have a problem with gambling? (Felt problem); Have people criticized your betting or told you that you had a gambling problem, regardless of whether or not you thought it was true? (Criticized); Have you felt guilty about the way you gamble or what happens when you gamble? (Felt guilty); Has gambling caused you any health problems, including stress or anxiety? (Health problem); Has your gambling caused any financial problems for you or your household? (Financial problem). For each item, respondents answered on a four-alternative scale $(0=$ never, $1=$ sometimes, 2 = most of the time, 3 = almost always). To examine the psychometric characteristics of PGSI in the present study, Exploratory Factor Analysis (EFA) and Parallel Analysis (O'Connor) were used to determine its appropriate number of factors. Reliability and Construct Validity of this instrument was detected using Reliability Analysis and Confirmatory Factor Analysis (CFA). The classification of adolescent gamblers into latent classes based on problem gambling severity levels was performed running a Latent Class Analysis. The optimal number of classes was determined by estimating models with an increasing number of classes, and then comparing those models using information criteria, classification criteria, the interpretability of each class, and the LRT tests, requesting TECH11 in the OUTPUT command of the Mplus syntax. Models that include between one and five latent classes were investigated for this purpose. Lower values of the Akaike information criterion (AIC), Bayesian information criterion (BIC), and adjusted BIC were preferred. The interpretation of the results was relied primarily on the BIC and the adjusted LRT. LRT tests the null hypothesis that a given model fits no better than a model with one less class. Failing to reject this test provides evidence for the model with one fewer class. There are used three covariates to increase the classification accuracy of adolescent gamblers into each latent class and to examine their influence on the outcome variable of problem gambling. These covariates which are already included in the model are: 1 . Gender, as binary categorical variable; 2 . Peer influence, as ordered categorical variable with four categories; 3 . Drug use, as ordered categorical variable with four categories. The scores were coded positively with high scores in the last two variables indicating higher frequency in gambling involvement and drug use, respectively.

\section{RESULTS}

The results from Parallel Analysis, using SPSS syntax (O'Connor, 2000) indicated the two-factor solution as the best solution for the PGSI measurement instrument. This is because only two factor eigenvalues listed under the heading "Prcntyle." (the $95^{\text {th }}$ percentile) are less than the factor eigenvalues (raw data column) from the original matrix (table1). Table 1: The results from Parallel Analysis

Root Raw Data Means Prcntyle 
1.0000004 .1587641 .1415921 .182471

2.0000001 .1866591 .0960911 .126727

3.000000 .7314581 .0590261 .084144

4.000000 .5774151 .0281331 .051004

5.000000 .554981 .9984371 .020402

Source: Authors' calculations.

The results from Exploratory Factor Analysis with one to three factors (EFA 13), using the robust estimator WLSMV and specifying the factor indicators as categorical in the Mplus syntax indicated that the two-factor model was the best fitting model in terms of fit and parsimony (table 2).

Table 2: Fit indices for EFA and CFA

\begin{tabular}{|l|l|l|l|l|}
\hline IC & One- factor model & Two-factor model & Three-factor model & $\begin{array}{l}\text { CFA } \\
\text { F1 by pgsi1-pgsi4 and F2 by pgsi5-pgsi9 }\end{array}$ \\
\hline$X^{2}$ & 343.934 & 57.444 & 22.384 & 103.994 \\
\hline RMSEA & 0.101 & 0.042 & 0.027 & 0.051 \\
\hline CFI & 0.956 & 0.995 & 0.999 & 0.989 \\
\hline TLI & 0.941 & 0.990 & 0.996 & 0.985 \\
\hline SRMR & 0.076 & 0.026 & 0.015 & - \\
\hline WRMR & - & - & - & 0.987 \\
\hline
\end{tabular}

Source: Authors' calculations.

The three-factor model resulted to have the best values of the fit indices, however, taking into account the parsimony of the model, the model with only two factors is considered to be the best solution. All the fit indices' values indicated a good fit of the model. The results of the table "Geomin Rotated Loadings" indicated that the first factor is highly correlated with the first four items (PGSI1-PGSI4) which assess "problem gambling behavior", whereas the second factor is highly correlated with the other five items (PGSI5-PGSI9) which assess "adverse consequences of gambling", as in table 3.

Table 3: Geomin rotated loadings

\begin{tabular}{|l|l|l|}
\hline PGSI items & Factor 1 & Factor 2 \\
\hline PGSI1 & 0.890 & -0.001 \\
\hline PGSI2 & 0.756 & 0.098 \\
& & \\
\hline PGSI3 & 0.544 & 0.299 \\
\hline PGSI4 & 0.790 & -0.014 \\
\hline PGSI5 & 0.201 & 0.588 \\
\hline PGSI6 & -0.081 & 0.749 \\
\hline PGSI7 & -0.015 & 0.838 \\
\hline PGSI8 & 0.013 & 0.809 \\
\hline PGSI9 & 0.058 & 0.724 \\
\hline
\end{tabular}

Source: Authors' calculations.

The correlation between the two factors is considerable $(r=0.685)$, indicating that both factors measure the same latent construct, the adolescent gambling problems. To evaluate the construct validity of this model, Confirmatory Factor Analysis (CFA) was performed. The values from all the fit indices indicated an acceptable fitting model (the last column of Table 2) which is evidence for an adequate construct validity In addition, to examine if the PGSI screening tool consistently reflect the one-dimensional construct that is measured, we have developed a Reliability Analysis. The Reability was calculated in terms of internal consistency for the entire scale (nine items) and separately for the two subscales, based on the two-factor solution.

PGSI resulted to have an adequate reliability in terms of internal consistency for the set of the nine items $(\alpha=0.853)$ and for the two subsets of items. Specifically, for the subset of the first four items (PGSI1-PGSI4) the coefficient of internal consistency is 0.785 , whereas for the second subset of indicators (PGSI5-PGSI9) this coefficient had a value $\alpha=0.808$. All the above results are a good evidence to believe that the measurement instrument PGSI is an adequate screening tool 
and measure only one latent construct, called "adolescent disordered gambling". The results from a Latent Class Analysis with one to five classes to classify adolescent gamblers into latent classes, based on their problem gambling severity levels identified with the PGSI screening tool, indicated that the model with three latent classes is considered the best solution. The best solution is evaluated while balancing the values of the information criteria, entropy, LRT $p$-value, theory, parsimony, interpretability, and average latent class probabilities. The three-class solution had the lowest BIC value, a relatively high entropy value, and a significant LRT test $p$-value (table 4).

Table 4: Fit indices for the appropriate latent class number model

\begin{tabular}{|l|l|l|l|l|l|l|}
\hline Nr. of classes & 1 & 2 & 3 & 4 & 5 & $\begin{array}{l}\text { 3+gender+peer } \\
\text { infl.+drugs }\end{array}$ \\
\hline Loglikelihood & -9243.350 & -8037.188 & -7821.598 & -7731.783 & -7658.304 & -7373.996 \\
\hline \# of parameters & 27 & 55 & 83 & 111 & 139 & 89 \\
\hline SCF for MLR & 1.000 & 1.042 & 1.094 & 1.082 & 1.135 & 1.091 \\
\hline AIC & 18540.700 & 16184.376 & 15809.196 & 15685.566 & 15594.609 & 14925.992 \\
\hline BIC & 18677.147 & 16462.323 & 16228.644 & 16246.514 & 16297.057 & 15373.027 \\
\hline SSABIC & 18591.386 & 16287.625 & 15965.009 & 15893.941 & 15855.548 & 15090.339 \\
\hline Entropy & NA & .867 & 0.828 & .775 & 0.787 & 0.848 \\
\hline $\begin{array}{l}\text { LMR-LRT p- } \\
\text { value }\end{array}$ & NA & .0000 & 0.0010 & 0.0863 & 0.3459 & 0.0000 \\
\hline $\begin{array}{l}\text { Most likely LC } \\
\text { membership \% }\end{array}$ & 100.00 & C\#1=36.73 & C\#1=13.31 & C\#1=12.36 & C\#1=9.33 & C\#1=11.23 \\
& C\#2=63.27 & C\#2=35.44 & C\#2=18.24 & C\#2=10.72 & C\#2=35.20 \\
& & C\#3=51.25 & C\#3=32.07 & C\#3=11.93 & C\#3=53.57 \\
\hline $\begin{array}{l}\text { C\#1 on gender, } \\
\text { peer infl. drugs }\end{array}$ & - & & C\#4=37.33 & C\#4=31.63 & C\#5=34.66 & \\
\hline $\begin{array}{l}\text { C\#2 on gender, } \\
\text { peer infl. drugs }\end{array}$ & - & - & - & - & - & 4.707 \\
\hline
\end{tabular}

\section{Source: Authors' calculations.}

The first class had a high average probability of endorsing all of the nine PGSI items, so this class is called "problem gamblers". The second class has moderate endorsement for those items. So this class is called "at-risk gamblers". The final class has low endorsement of all items and comprised the largest percentage of the sample and so it is called the "non-problem gamblers" class (Figure 1.).

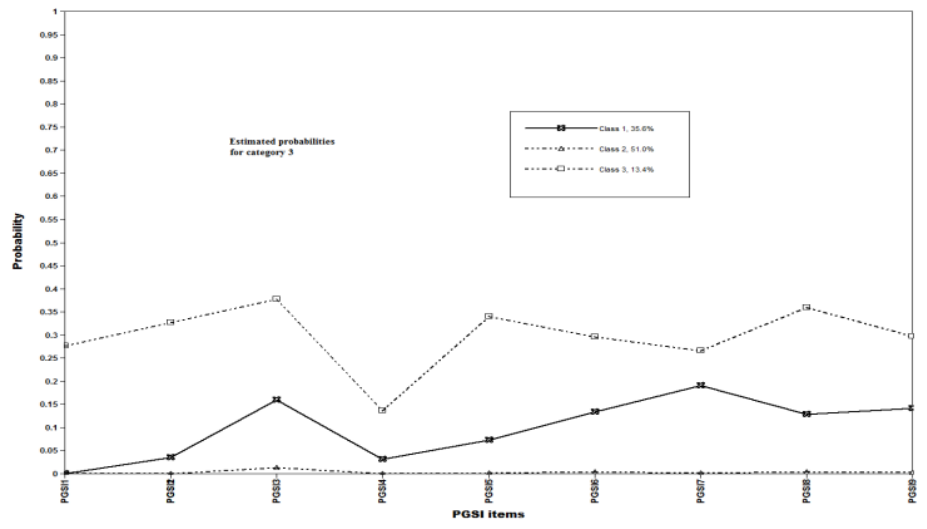

Source: Authors' calculations.

Figure 1. Estimated probabilities of latent classes for category 3 of PGSI 
The entropy is 0.828 , while $13.31 \%$ of adolescent gamblers are classified as "problem gamblers", $35.44 \%$ are classified as "at-risk gamblers", whereas $51.25 \%$ are classified as "non-problem gamblers". A slight shift is caused by the simultaneously inclusion of three covariates (gender, peer influence and substance use) into the three-class model. However, the latent class model with covariates is improved and the odds ratios' values from a Logistic Regression indicated that adolescent males are over 4 times and about 3 times more likely to belong to the "problem gamblers" and "at-risk gamblers" class respectively, compared to females, holding the "non-problem gamblers" class as reference class. Adolescent gamblers who used substances are over 17 and 5 times more prone to belong to "problem gamblers" and "atrisk gamblers" class respectively, compared to non-users, taking the "non-problem gamblers" class as reference class. Finally, having peers who gamble increases the probability to belong to "problem gamblers" and "at-risk gamblers" class. However, the influence of the three covariates is not statistically significant for the three latent classes

\section{CONCLUSIONS}

The study indicated that PGSI may be an adequate measuring instrument to identify and assess adolescent problem gambling severity levels. This measurement instrument resulted to have satisfactory psychometric properties measuring only one latent construct, called "adolescent disordered gambling". Latent Class Analysis, specifying the indicators as categorical and using robust estimators, may be an adequate method for identifying problem gambling severity levels for cross-sectional data, non-normally distributed. Subtyping adolescent gamblers may improve our understanding of the etiology of problem gambling. The high prevalence rates of adolescent "problem gamblers" and "at-risk gamblers" indicate that, the involvement of the adolescents in gambling may be associated with many problems for their future.

\section{REFERENCES}

[1] Akaike, H. (1987). Factor analysis and AIC. Psychometrika, 52, 317-332.

[2] American Psychiatric Association (APA) (2013).Diagnostic and Statistical Manual of Mental Disorders (DSM-5). Arlington, VA: American Psychiatric Publishing.

[3] Asparouhov Tihomir and Muthen Bengt (2014). Auxiliary Variables in Mixture Modeling: Using the BCH Method in Mplus to Estimate a Distal Outcome Model and an Arbitrary Secondary Model Mplus Web Notes: No. 21 Version 2 October 7.

[4] Barnes, G. M., Welte, J. W., Hoffman, J. H., \& Tidwell, M.-C. O. (2009). Gambling, alcohol,and other substance use among youth in the United States. Journal of Studies on Alcohol and Drugs, 70, 134-142.

[5] Bentler, Peter M. (1988). Comparative fit indexes in structural models. Psychological Bulletin, 107, 238246.

[6] Blanco C, Hasin DH, Petry N, Stinson FS, Grant B (2006). Sex differences in subclinical and DSMIV pathological gambling: results from the National Epidemiological Survey on Alcohol and Related Conditions. Psychological Medicine; 36: 943-953.

[7] Browne, Michael W., Cudeck Robert (1993). Alternative Ways of Assessing Model Fit. In: Bollen, Kenneth A., Long, J. Scott. editors. Testing Structural Equation Models. Sage; Newbury Park, CA, 136-162.

[8] Buchta, R. M. (1995). Gambling among adolescents. Clinical Pediatrics, 34(7), 346-348.

[9] Chiu, J., \& Storm, L. (2010). Personality, perceived luck and gambling attitudes as predictors of gambling involvement. Journal of Gambling Studies, 26, 205-227.

[10] Collins LM, Fidler PL, Wugalter SE, Long JD. (1993). Goodness-of-fit testing for latent class models. Multivariate Behavioral Research. 28(3):375-389.

[11] Collins, L.M., and Lanza, S.T. (2010). Latent class and latent transition analysis: With applications in the social, behavioral, and health sciences. New York: Wiley.

[12] Daghestani, A. N., Elenz, E., \& Crayton, J. W. (1996). Pathological gambling in substance Abusing veterans. Journal of Clinical Psychiatry, 57, 360-363.

[13] Dayton, C.M., and Macready, G.B. (1988). Concomitant-variable latent-class models. Journal of the American Statistical Association, 83, 173-178. 
[14] Derevensky, J. L., Sklar, A., Gupta, R., \& Messerlian, C. (2010). An empirical study examining the impact of gambling advertisements on adolescent gambling attitudes and behaviors. International Journal of Mental Health and Addiction, 8, 21-34.

[15] Dickson LM, Derevensky JL, Gupta R (2008). Youth gambling problems: examining risk and protective factors. Int Gambl Stud;8(I):25-47.

[16] Donati MA, Chiesi F, Primi C (2012). A model to explain at-risk/problem gambling among male and female adolescents: gender similarities and differences. J Adolesc.; 35:129-37. doi: 10.1016/j.adolescence.2012.10.001.

[17] Ferris, J., \& Wynne, H. (2001). The Canadian problem gambling index: Final report. Submitted for the Canadian Centre on Substance Abuse.

[18] Goldstein, A. L., Walton, M. A., Cunningham, R. M., Resko, S. M., \& Duan, L. (2009). Correlates of gambling among youth in an inner-city emergency department. Psychology of Addictive Behaviors, 23, 113-121.

[19] Goodman, L. A. (1974). Exploratory latent structure analysis using both identifiable and unidentifiable models. I, 2, 215-231.

[20] Griffiths, M. (1991). The observational study of adolescent gambling in UK amusement arcades. Journal of Community \& Applied Social Psychology, 1(4), 309-320.

[21] Gupta R, Derevensky JL (2000). Adolescents with gambling problems: from research to treatment. J Gambl Stud; 16 (2/3):315-42.

[22] Hagenaars JA, McCutcheon AL. (2002). Applied latent class analysis. Cambridge University Press; Cambridge.

[23] Hardoon KK, Derevensky JL (2002). Child and adolescent gambling behaviour: current knowledge. Clin Chil Psych and Psychiatr.; 7:263-81.

[24] Horn, J.L. (1965). A rationale and test for the number of factors in factor analysis. Psychometrika, 30(2): 179-185.

[25] Ibanez A, Blanco C, Moreryra P, Saiz-Ruiz J (2003). Gender differences in pathological gambling. Journal of Clinical Psychiatry; 64: 295-301.

[26] Jackson, A. C., Dowling, N. A., Thomas, S. A., Bond, L., \& Patton, G. (2008). Adolescent gambling behaviour and attitudes: A prevalence study and correlates in an Australian population. International Journal of Mental Health and Addiction, 6, 325-352.

[27] Jackson, A. C., Wynne, H., Dowling, N. A., Tomnay, J. E., \& Thomas, S. (2009). Using the CPGI to determine problem gambling prevalence in Australia: Measurement issues. International Journal of Mental Health and Addiction. doi: 10.1007/s11469-009-9238-9

[28] Jacobs, D. F. (2000). Juvenile gambling in North America: An analysis of long-term trends and future prospects. Journal of Gambling Studies, 16, 119-152.

[29] Jedidi, K., Jagpal, H., \& DeSarbo W. S. (1997). Finite-mixture structural equation models for responsebased segmentation and unobserved heterogeneity. Marketing Science, 16, 39-59.

[30] Joreskog, Karl G., \& Sorbom, Dag (1981). LISREL V: Analysis of linear structural relationships by the method of maximum likelihood. Chicago: National Educational Resources.

[31] Ladd GT, Petry NM (2002). Gender differences among pathological gamblers seeking treatment. Exper Clinical Psychopharmacol; 10: 302-309.

[32] Lazarsfeld, P. F. and Henry, N. W. (1968). Latent Structure Analysis. Houghton Mifflin.

[33] Magidson, J. And Vermunt. J. (2004). Latent class models. In The SAGE Handbook of Quantitative Methodology for the Social Sciences, D. Kaplan, Ed. Sage Publications, Thousand Oaks, CA, 175-198. 
[34] McCutcheon, A. L. (1987). Latent class analysis. Quantitative Applications in the Social Sciences Series 64. Sage Publication, Thousand Oaks, California.

[35] McMillen, J., \& Wenzel, M. (2006). Measuring problem gambling: Assessment of three prevalence screens. International Gambling Studies, 6(2), 147-174. doi: 10.1080/14459790600927845

[36] Muthén Linda K., Muthén Bengt O. (1998-2001). MPlus User's Guide, 2nd Version. Muthén \& Muthén; Los Angeles.

[37] Nylund KL, Asparouhov T, Muthén B. (2007). Deciding on the number of classes in latent class analysis and growth mixture modeling: A Monte Carlo simulation study.Structural Equation Modeling: A Multidisciplinary Journal.14(4):535-569.

[38] O'Connor, B. P. (2000). SPSS and SAS programs for determining the number of components using parallel analysis and Velicer's MAP test. Behavior Research Methods, Instruments, \& Computers, 32, 396-402.

[39] Petry N.M (2000). Psychiatric symptoms in problem gambling and non-problem gambling substance abusers. Am J Addict; 9(2): 163-71.

[40] Petry, N.M. (2005). Pathological Gambling. Etiology, Comorbidity, and Treatment. Washington, DC: American Psychological Association.

[41] Potenza MN, Steinberg MA, Wu R, Rounsaville BJ, O'malley SS (2006). Characteristics of Older Adult Problem Gamblers Calling a Gambling Helpline. Journal of Gambling Studies; 22: 241-254.

[42] Schwartz, G. (1978). Estimating the dimension of a model. The Annals of Statistics, 6, 461-464.

[43] Sclove, L. S. (1987). Application of model-selection criteria to some problems in multivariate analysis. Psychometrika, 52, 333-343.

[44] Shaffer, H. J., Hall, M. N., \& Vander Bilt, J. (1999). Estimating the prevalence of disordered gambling behavior in the United States and Canada: A research synthesis. American Journal of Public Health, 89, 1369-1376.

[45] Svetieva, E. \& Walker, M. (2008). Inconsistency between concept and measurement: The Canadian Problem Gambling Index (CPGI). Journal of Gambling Issues, 22. Available: http://www.camh.net/egambling/issue22/pdfs/01svetievawalker.pdf. Accessed July 19, 2010

[46] Tavares H, Zilberman ML, Bites FJ, Gentil V (2001). Gender differences in gambling progression. Journal of gambling Studies; 17: 151-159.

[47] Tucker, Ledyard R. and Lewis, Charles (1973). A reliability coefficient for maximum likelihood factor analysis. Psychometrika, 38, 1-10.

[48] Vermunt J. K., Magidson J. (2005). Structural equation models: mixture models, in Encyclopedia of Statistics in Behavioral Science, eds Everitt B., Howell D., editors. (Chichester: Wiley; ), 1922-1927 10.1002/0470013192.bsa600.

[49] Vermunt, Jeroen K. (2010). "Latent Class Modeling with Covariates: Two Improved ThreeStep Approaches." Political Analysis 18:450-469.

[50] Wurpts I. C. and Geiser C (2014). Is adding more indicators to a latent class analysis beneficial or detrimental? Results of a Monte-Carlo study. Front. Psychol., 21 August 2014

[51] http://dx.doi.org/10.3389/fpsyg.2014.00920.

[52] Yang, C. (2006). Evaluating latent class analyses in qualitative phenotype identification. Computational Statistics \& Data Analysis, 50, 1090-1104.

Emil FRASHERI has graduated the Faculty of Economy in Tirana in 1990 with profile in Economical Statistics. Since 2012, he is a lecturer in the Department of Management in the Faculty of Economics in Fan .S. Noli University of Korça. $\mathrm{He}$ is also a Phd candidate in the Department of Mathematics, Informatics and Applied Statistics in the Faculty of Economy of Tirana. It is the beginning of his scientific research work. His work is focused on the statistical analyses of gambling related problems. 